\title{
La consolation dans les tombeaux poétiques en Italie, en France et dans les Îles britanniques (1590-1640)
}

\section{Paule Desmoulière}

\section{(2) OpenEdition Journals}

Édition électronique

URL : http://journals.openedition.org/rhetorique/525

DOI : $10.4000 /$ rhetorique. 525

ISSN : 2270-6909

Éditeur

UGA Éditions/Université Grenoble Alpes

Édition imprimée

ISBN : 978-2-37747-010-5

\section{Référence électronique}

Paule Desmoulière, "La consolation dans les tombeaux poétiques en Italie, en France et dans les îles britanniques (1590-1640) », Exercices de rhétorique [En ligne], 9 | 2017, mis en ligne le 21 juin 2017, consulté le 12 septembre 2020. URL : http://journals.openedition.org/rhetorique/525 ; DOI : https:// doi.org/10.4000/rhetorique.525

Ce document a été généré automatiquement le 12 septembre 2020.

\section{c) (1) (2)}

Les contenus de la revue Exercices de rhétorique sont mis à disposition selon les termes de la Licence Creative Commons Attribution - Pas d'Utilisation Commerciale - Partage dans les Mêmes Conditions 4.0 International. 


\title{
La consolation dans les tombeaux poétiques en Italie, en France et dans les Îles britanniques (1590-1640)
}

\author{
Paule Desmoulière
}

1 Dans la philosophie antique, la parole apparaît comme un médecin qui soigne les souffrances de l'âme. Ainsi, au début de la consolation à Apollonius, Plutarque dit que "le discours est un bon médecin pour l'âme malade, si on le tient au bon moment pour apaiser un cœur » («Psukhès nosousès eisin iatroi logoi, hotan tis en kairô ge malthassè ${ }_{i}$ kear $^{1}$ »). En particulier, l'orateur et le philosophe sont des interlocuteurs privilégiés pour ceux qui sont en deuil ou qui redoutent leur propre mort. Après l'Antiquité, ce rôle de consolateur fut repris par les pères de l'Église (Ambroise, Jérôme, Paulin de Nole) ainsi que par les humanistes comme Pétrarque, souvent sous la forme du sermon ou de la lettre.

2 Qu'en est-il du poète ? La parole poétique est-elle également vue comme un moyen de consoler ceux qui sont en deuil ? On trouve des exemples de discours consolatoire dans la poésie latine de l'Antiquité, chez Lucrèce, dans les Pontiques d'Ovide, les Silves de Stace et la Consolatio ad Liviam du pseudo-Ovide ${ }^{2}$. On en trouve aussi des traces chez certains poètes élégiaques ${ }^{3}$, ainsi que dans les Odes d'Horace ou les épigrammes de Martial ${ }^{4}$.

3 Ces sources étaient connues et citées par les auteurs de la Renaissance, donc il n'est guère étonnant que le discours de consolation ait également été très présent dans la poésie des $\mathrm{XVI}^{\mathrm{e}}$ et $\mathrm{XVII}^{\mathrm{e}}$ siècles, comme en témoigne la célèbre "Consolation à M. Du Perier» de Malherbe. Pourtant, alors que les dialogues et les lettres de consolation de cette période ont fait l'objet d'études, le corpus des consolations en vers est très peu étudié. Cet article se propose donc d'évoquer la présence de la consolation dans les tombeaux poétiques imprimés en Italie, en France et dans les Îles britanniques entre les années 1590 et $1640^{5}$. 
Cela permet de poser deux questions : quelle est la place de la consolation dans les autres discours de commémoration des défunts, notamment dans la triade composée de la lamentation, de l'éloge et de la consolation? Quelle place occupe la consolation dans les tombeaux poétiques et quel rôle ils jouent dans ces recueils collectifs?

\section{Comment définir la consolation?}

5 Si l'on veut définir la consolation, il faut constater, à la suite de Giuseppe Chiecchi ${ }^{6}$, qu'elle est un type de discours plutôt qu'un "genre" au sens où ce terme est habituellement utilisé (un élément de discours défini aussi bien par des critères formels que par un type de contenu). En effet, la consolation peut prendre la forme d'un discours en prose, comme une lettre, un sermon, un dialogue philosophique (par exemple chez Boèce), mais peut tout aussi bien être exprimée en vers, dans une élégie, des stances ou un sonnet. Dès lors, comment définir ce qu'est une consolation? Il n'existe sans doute pas de définition exhaustive du terme, mais on peut du moins affirmer que la consolation est un type de discours qui se distingue par la combinaison entre une situation d'énonciation particulière et un sujet ou thème. De façon générale, donc, on peut affirmer que la consolation est un discours dans lequel un énonciateur individuel ou collectif s'adresse à un destinataire pour lui apporter une forme de réconfort. Dans le cadre des tombeaux poétiques, ce thème est encore plus précis, puisqu'il s'agit spécifiquement de consolatio mortis, visant à soulager le chagrin du deuil.

Une fois cette définition posée, reste à savoir quelle place occupe la consolation dans la nébuleuse des discours de commémoration des défunts. On peut trouver des éléments de réponse en confrontant les réflexions proposées par les traités de rhétorique de la Renaissance aux pratiques d'écriture de la consolation dans les tombeaux poétiques.

\section{Le rapport de la consolation avec d'autres formes de discours funèbres}

7 Pour répondre à la question de la place de la consolation dans le discours de commémoration des défunts, il peut être utile de considérer comment le discours de consolation apparait dans les tombeaux poétiques. Quel est le rapport de la consolation avec la lamentation? Apparaît-elle systématiquement après une lamentation? La consolation prend-elle la forme d'une élégie, d'une épitaphe, d'une nénie ou d'un sonnet? Pourquoi utilise-t-on une forme ou une appellation plutôt qu'une autre? Pourquoi certains poèmes sont-ils intitulés «consolation » et d'autres non? Cela pose bien entendu le problème plus vaste des usages des titres et des traditions littéraires dans le corpus des tombeaux poétiques.

Dans sa Poétique, Jules-César Scaliger définissait la consolation comme l'une des cinq parties de l'épitaphe, en affirmant que « les parties de l'épitaphe occasionnelle sont les suivantes: l'éloge, l'évocation de l'ampleur de la perte subie, la lamentation, la consolation, l'exhortation ${ }^{7}$ ». Ménandre le rhéteur traite quant à lui des discours funèbres sous trois aspects : l'oraison funèbre (epitaphios logos), la monodie (monôdia) ou lamentation funèbre et la consolation (paramuthètikos logos) et il les classe dans la catégorie des discours épidictiques écrits à la louange d'un homme ${ }^{8}$. La question qui se 
pose dès lors est celle du rapport entre les consolations des tombeaux poétiques et ces autres formes de célébrations posthumes.

Scaliger et Ménandre suggèrent donc que la consolation constitue un aboutissement de l'éloge funèbre, un discours qui suit généralement la lamentation. De fait, un certain nombre de poèmes que l'on trouve dans les tombeaux poétiques suivent cette évolution de la lamentation à la consolation.

On peut donner l'exemple d'une nénie qui se trouve dans un recueil imprimé en 1611 par le collège jésuite de la Flèche suite aux commémorations anniversaires pour la mort d'Henri IV ${ }^{9}$. Ce poème est scandé par une injonction aux larmes.

Allons, fais sortir les ruisseaux de tes yeux, France, revêts des habits de deuil, Arrache, France, tes cheveux et lacère tes joues.

Duc rivos oculis, pullatas Gallia vestes

Indue, rumpe comam Gallia, rumpe genas ${ }^{10}$.

11 Ce refrain apparait trois fois au cours du poème et alterne avec des vers anaphoriques qui commencent par la formule «Vixit qui » (« il a vécu, celui qui » etc.). À la fin du poème, cependant, une nouvelle anaphore apparaît: «Si fles» («si tu pleures»). Ce changement marque le passage de la lamentation à la consolation, puisque le distique qui intervient alors deux fois à la fin du poème exhorte désormais la France non plus à pleurer, mais au contraire à se défaire de sa posture de deuil.

Je suis trompé ; essuie tes yeux, France, quitte ces habits de deuil ;

Épargne ta chevelure, France, épargne tes joues.

Fallor; terge oculos, pullatas Gallia vestes

Exue; parce comae Gallia, parce genis ${ }^{11}$.

On retrouve souvent ce mouvement de la lamentation à la consolation dans les poèmes des tombeaux poétiques intitulés "épicèdes». Cela s'explique par le fait que les épicèdes antiques et humanistes, comme celles de Stace ou de Politien, suivent souvent ce type de progression ${ }^{12}$. Dans son commentaire des Silves, par exemple, José Esteve Forriol a montré comment les épicèdes de Stace comportent une introduction, une laudatio, avec ou sans comploratio, une description de la maladie finale du défunt et enfin une consolatio ${ }^{13}$.

Les poèmes intitulés "nénies " peuvent également suivre cette progression de la lamentation à la consolation. C'est le cas de la nénie pour la mort du médecin Simon de Provenchères : dans les quatre premiers vers, le poète rappelle la mort de monsieur de Provenchères à Paris en 1617, loin de sa ville natale de Sens. Ensuite, le poète fait la louange du défunt, autant pour ses qualités de médecin («Par fuit Antiquis homines sanare peritis »: "Il fut l'égal des anciens habiles dans l'art de soigner »), que pour la constance de son esprit et pour ses mérites personnels ("egregius civis, prudensque, piusque » : " concitoyen illustre, prudent et pieux »). Cet éloge s'achève sur le topos des hommes bons qui partent prématurément au Paradis. Suit enfin la consolatio, qui suit les thèmes traditionnels de la consolation chrétienne: le poète enjoint les proches du défunt de retenir leurs lamentations, parce que ces larmes ne sont pas dignes d'un chrétien, dans la mesure où leur parent est au Ciel ${ }^{14}$.

14 Pourtant, la lamentation, l'éloge et la consolation ne se succèdent pas toujours selon un ordre immuable et les poètes adaptent et modifient souvent l'articulation des ces trois discours selon leurs besoins.

En outre, dans les titres des poèmes eux-mêmes, la consolation n'est pas toujours clairement distinguée des autres formes de commémoration des défunts, comme 
l'épitaphe. Ainsi, dans un recueil imprimé à Aberdeen en 1634 à la mémoire de William Michel, fils d'un pasteur de la ville, l'on trouve une lettre de consolation adressée à la mère du défunt intitulée "An epitaph or consolatory epistle ${ }^{15}$ ». Par ce titre, l'auteur précise bien qu'il s'agit d'une consolation et prend soin de rappeler les origines épistolaires de ce type de discours. Toutefois, il est intéressant de noter que l'auteur conçoit également que la consolation puisse aussi être intitulée « épitaphe ".

Cette association de l'épitaphe et de la consolation reste relativement rare dans les tombeaux poétiques, peut-être parce que les poètes tendent à associer l'épitaphe à une brève inscription gravée sur la tombe. Comme on l'a vu, Scaliger suggère que la consolation peut également constituer une partie de l'épitaphe, le discours prononcé en l'honneur du défunt. D'ailleurs, dans une note qu'il ajoute à son poème, le pasteur qui a composé l'épitaphe-épître consolatoire sur la mort de William Michel précise qu'il s'appuie une tradition patristique :

On appelait des oraisons et des épîtres de cette nature épitaphes, il y a fort longtemps, comme un chacun peut le voir dans les oraisons de Nazianze et les épîtres de Jérôme sur le même sujet.

Orations and Epistles of this nature, were called Epitaphs, manie ages since, as any may perceave by Nazianens Orations, and Hieroms Epistles, of the lyke subject ${ }^{16}$.

On voit donc que la consolation occupe une place relativement extensible au sein des différents discours à la mémoire des défunts, qui résulte en une pratique poétique assez variée. On observe la même variété dans les formes poétiques que peut revêtir la consolation.

\section{Les formes de la consolation}

En effet, étant donné que la consolation n'est pas définie par des critères formels, elle peut revêtir une multiplicité de formes poétiques et se mêler à d'autres traditions de commémoration funèbre. Par conséquent, alors qu'on pourrait considérer la consolation comme un discours argumentatif relativement long adapté à une forme comme les stances, la longueur des formes poétiques choisies peut varier considérablement. Ainsi, le discours de consolation peut être exprimé dans des formes brèves d'origine lapidaire comme l'épitaphe ou l'épigramme.

En effet, la consolation peut être longue et argumentative, mais également fort brève et plus virtuose que philosophique, comme cette consolation anagrammatique d'une ligne pour la mort de Charlotte du Gué intitulée :

NICOLAUS E VERDUNO ET CAROLA DU GUAI. Anagrammatice.

Vivus dolor! gaude, en cara tua in caelo ${ }^{17}$.

Nicolas de Verdun et Charlotte du Gué. Anagramme.

Vivante douleur ! Réjouis-toi donc, voici au ciel celle qui t'es chère.

20 Le choix d'écrire une consolation sous une forme brève permet au poète de faire preuve de sa virtuosité poétique et rhétorique. Dans l'épigramme, par exemple, le poète est obligé d'exprimer les arguments habituels de consolation dans un nombre de vers très limité. Dans un recueil imprimé à Vicence en 1612 à la mémoire du comte Jacopo Valmarana, l'on trouve une épigramme de consolation qui prend la forme d'une prosopopée du défunt, comme l'indique son titre: "Epigramma. Matrem suam lacrymantem Iacobus moriens consolatur ${ }^{18}$ " ("Épigramme. Jacopo mourant console sa mère qui pleure toutes ses larmes »). Pourtant, malgré la brièveté de la forme, on retrouve dans ce poème les étapes habituelles du discours de consolation: le 
questionnement de la valeur des larmes (« Pourquoi fonds-tu en larmes, parce que le chagrin accable ton cœur, / Ô mère, part et moitié de mon âme ${ }^{19}$ ? »), l'injonction à les sécher ( «Si tu m'aimes, ô mère, retiens tes pleurs qui coulent, / Je t'en prie, délivre ton cœur d'une languissante douleur $\left.{ }^{20} »\right)$ et la négation de la réalité de la mort ( «Je ne mourrai pas, laissant quelque chose de mortel aux funérailles, / Je vivrai : plutôt que de penser que je suis mort, pense que je m'en suis alléé $\left.{ }^{21}\right)$.

21 Si les consolations sont souvent des poèmes brefs, on en trouve des plus longues, notamment en France à partir des années 1620. Ainsi, les consolations de Marchant et Mercier à Cléante-Brun sur la mort de son Alcinde font chacune plus d'une quarantaine de vers ${ }^{22}$. Le " poème consolatif » adressé par le doyen Jean Le Vasseur à l'archidiacre de Nyon sur la mort de son frère Claude de Montigny en 1625 fait plus d'une centaine de vers ${ }^{23}$.

En fin de compte, les auteurs des consolations se laissent une assez large marge de manœuvre pour choisir la forme que prend la consolation (le choix du mètre et les associations avec d'autres formes de commémorations funèbres). Cette fluidité est un avantage, dans la mesure où elle laisse aux auteurs des vers la possibilité de choisir ce qui convient le mieux à la fois aux défunts qu'ils célèbrent et au public auquel ils s'adressent ${ }^{24}$.

Constatant cette variété, on est en droit de se demander sur quels critères on peut s'appuyer pour définir la consolation. On peut trouver un premier élément de réponse dans un thème topique que l'on retrouve dans presque toutes les consolations poétiques : l'évocation initiale des larmes de celui qui doit être consolé.

\section{Les larmes et la concessio doloris}

Bien que les destinateurs et les destinataires soient assez variés, on observe que la progression du discours dans ces poèmes de consolation tend à suivre une même logique : les consolations s'ouvrent généralement sur une évocation des larmes de ceux qui sont en deuil, souvent réprouvées par le consolateur, puis la réalité de la mort est niée au profit de l'immortalité du défunt dans l'au-delà. Si, dans les consolations en prose, les arguments peuvent varier en fonction de l'éthos du consolateur et du destinataire, les consolations en vers sont généralement moins argumentées, en partie parce qu'elles sont souvent relativement brèves (moins d'une vingtaine de vers). On peut cependant identifier dans les consolations en vers un certain nombre d'arguments et des lieux qui reviennent fréquemment.

En particulier, l'on peut identifier un certain nombre de lieux communs autour de la question des larmes de celui qui est en deuil. L'un des éléments qui revient le plus souvent dans la consolation de façon générale est la concessio doloris, ce moment où le consolateur admet que la douleur de celui qui console est légitime. Giuseppe Chiecchi a montré que, depuis Ambroise, les larmes sont reconnues comme une étape inévitable de la consolation chrétienne ${ }^{25}$.

Dans les consolations des tombeaux poétiques, on observe que l'évocation des larmes fait véritablement figure de passage obligé. En effet, presque toutes les consolations des recueils mentionnent les larmes de celui qui est en deuil, généralement au début du texte. En ce sens, l'on peut dire que l'évocation des larmes est plus qu'une image du deuil, c'est ce qui marque l'ouverture de la rhétorique consolatoire : pour mettre en 
mouvement cette rhétorique, il semble nécessaire de faire un constat initial de la douleur que la consolation vise à dissiper par l'argumentation ou par la persuasion. À partir de là, la consolation s'organise la plupart du temps en deux mouvements opposés et dialectiques: la concessio doloris et la refutatio, appuyées sur des exempla. La consolation s'achève alors par une affirmation de l'inutilité de la douleur, par le biais d'un argument ou d'une sentence.

En pratique, les poètes varient beaucoup autour de ce schéma. Ils ne l'appliquent pas strictement et les étapes topiques de ce discours peuvent changer de place selon l'identité de la personne à laquelle le discours est adressé. Par exemple, l'évocation des larmes se présente souvent en début de texte, sous la forme d'une injonction qui commande au destinataire de cesser de pleurer. Ainsi, dans le Temple d'honneur imprimé en 1622 à la mémoire du baron de Frican, à l'initiative d'Adam Scaliger, on trouve plusieurs consolations qui commencent de cette façon : « Relasche tes soupirs », dans la pièce intitulée L'ombre du baron de Frican au chevalier de L'Escale, ou encore «Bornez vos pleurs l'Escale", tiré des Stances sur le même ${ }^{26}$. Cependant, les phrases évoquant les larmes ne sont ni toujours injonctives, ni systématiquement placées en début de texte. Il arrive par exemple que la consolation s'ouvre sur la représentation d'un personnage en larmes. Une consolation publiée dans le tombeau de Catherine de Rohan, duchesse de Deux-Ponts, s'ouvre ainsi sur un tableau mythologique. Zeus y apparait, pleurant la mort de Sémélé :

C'est ainsi que le fils de Rhée

Par une cause inespérée

Soumis à la rigueur du sort

Pleura la disgrâce inhumaine

De cette princesse Thébaine

À qui son feu donna la mort ${ }^{27}$.

La comparaison entre Zeus/le duc de Deux-Ponts et Sémélé/Catherine est explicitée un peu plus loin :

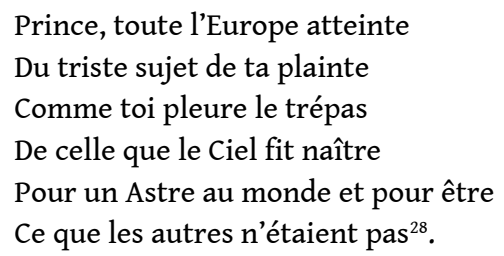

La consolation elle-même est divisée en trois parties : une première section développe cette allusion mythologique, la deuxième section est adressée au mari de la défunte et la dernière à sa mère. L'argument utilisé pour consoler le mari est le topos de la beauté trop belle pour la terre, enlevée par le ciel, où l'intertexte de Malherbe est patent :

Et parmi les choses mortelles

C'est un malheur que les plus belles

Ne durent pas plus d'un matin ${ }^{29}$.

L'argument employé pour consoler la mère de la défunte est en revanche son immortalité au ciel. Ce texte reprend des arguments de la consolation patristique, puisque le poète suggère que les larmes de la mère sur le passage de sa fille dans l'audelà pourraient être considérées comme impies. Dans la section du poème adressée au mari de la duchesse, c'est la beauté de l'épouse qui est louée et dans celle adressée à sa mère, ses vertus chrétiennes. Le poète suit en réalité une progression usuelle de la consolation, en passant de l'évocation de la beauté physique de la défunte à ses qualités morales et, partant, à son immortalité, mais il a choisi de mettre en relief cette 
progression en s'adressant à chaque fois au destinataire le plus propre à recevoir ces arguments. Cette consolation a le mérite de mettre en relief à quel point le destinataire de la consolation est un interlocuteur important dans le développement de l'argumentation consolatoire. Là encore, la liberté de choix qu'offre la consolation vise à son efficacité rhétorique, puisqu'elle permet aux poètes d'adapter le discours en fonction de la situation d'énonciation du discours.

Une possibilité de définir plus précisément les contours de la consolation en vers semble dès lors se profiler: il s'agirait d'une forme de discours adressée à un interlocuteur qui ne prend pas directement la parole (une sorte de dialogue dont on n'entendrait qu'une partie, en somme). Cependant, les modalités de ce dialogue varient en fonction de l'éthos du consolateur et de consolé.

\section{Un discours adressé : diversité des destinataires}

La consolation suppose en effet la participation d'au moins deux voix, trace de son origine épistolaire ou de son lien avec le dialogue philosophique, sans doute. Alexandre Tarrête a insisté sur le fait que cette forme dialogique correspondait bien aux tensions inhérentes à la consolation, car celle-ci nécessite un dialogue :

[...] on ne saurait imaginer de consolation véritable dans la solitude. Le dialogue avec une forme d'altérité est ici nécessaire: ami, parent, prochain, hommes ou dieux apportent à celui qui souffre réconfort et exhortation. [...] Dans tous les cas, la médiation d'autrui est nécessaire pour rapprocher l'âme souffrante de la sagesse ${ }^{30}$.

La consolation est donc toujours un discours adressé à quelqu'un. À qui s'adresse-t-on ? Dans les tombeaux poétiques, la plupart des consolations sont adressées à un proche parent du défunt. Il peut s'agir du père, de la mère ou encore du conjoint du défunt. Il arrive même qu'une consolation soit adressée à plusieurs personnes à la fois, comme dans la «Consolation à Monsieur le Duc de Deux-Ponts \& Madame de Rohan sur la mort de Madame la Duchesse de Deux-Ponts ${ }^{31}$ ». La mère du défunt reste cependant une destinataire privilégiée du discours de consolation, comme c'était déjà le cas dans les lettres de Sénèque ${ }^{32}$. La position sociale du défunt peut également avoir une influence sur le choix du destinataire. Quand le défunt est de rang royal, l'on s'adresse à ses parents à la fois en tant que proches du défunt et en tant que souverains. Les deux sonnets de consolation adressés à Philippe II par Giovanni Battista Basile et Arcangelo Spina sont intitulés "Consola la maestà del $R e^{33}$ ».

Par ailleurs, quand le défunt est de sang royal ou qu'il occupe des fonctions officielles, il arrive souvent que l'entité à laquelle on s'adresse soit un personnage allégorique, très souvent le pays ou la ville du défunt, sorte de parent symbolique. Cet élément est également relativement topique, comme Gerardus Vossius l'a remarqué dans son traité de poétique ${ }^{34}$. Par exemple, c'est « Gallia » qui est consolée à l'occasion du décès d'Henri $\mathrm{IV}^{35}$, mais aussi après celle du cardinal de Richelieu ${ }^{36}$. Bologne en pleurs est consolée lors du décès de Carlo Barberini ${ }^{37}$. De même, c'est le peuple britannique en pleurs qui est consolé, en 1613, après la mort du fils aîné de Jacques I ${ }^{\mathrm{er}}$, Henry, prince de Galles ${ }^{38}$. Dans certains contextes politiques, le poète s'adresse plutôt à ses concitoyens, comme les citoyens de Viterbe sont consolés de la mort de leur gouverneur Enea Vaini ${ }^{39}$. Dans d'autres cas, c'est la famille du défunt qui réconforte la ville, comme dans ce poème où la famille d'Adriano Baglioni console la ville de Pérouse ${ }^{40}$. Enfin, dans les recueils d'Oxford et de Cambridge, l'on adresse parfois la consolation à l'université (« academia 
»), comme dans les Justa sur la mort de Thomas Bodley, où l'on trouve un poème « Ad Academiam Carmen consolatorium ${ }^{41}$ ". Là encore, les choix sont faits non en fonction de critères formels rigides, mais selon la condition du défunt et le public qui est concerné. Ainsi, la consolation pour Thomas Bodley, qui figure dans un recueil composé à la mémoire du fondateur de la célèbre bibliothèque bodléienne par des membres de l'université d'Oxford (donc à la gloire de cette institution) ne pouvait qu'être adressée à l'academia elle-même.

Enfin, le consolateur lui-même se présente sous des traits assez variés. Il peut parler en tant qu'ami du défunt, mais également comme un inférieur hiérarchique dont le devoir est de consoler son mécène.

Le consolateur peut par exemple présenter une consolation à un mécène ou à un protecteur. Dans ce cas, l'enjeu n'est pas le même pour le poète. La consolation intitulée "À l'excellent I.V.D.D. Giulio Volpi son illustre mécène. Sur la mort du Conte Luigi Porto, consolation d'un protégé reconnaissant» est explicitement adressée à un mécène ${ }^{42}$. Le poème lui-même est particulièrement travaillé, puisqu'il s'agit d'un double acrostiche, où l'on peut lire, à droite du texte : «Aloisio Porto » et à gauche «Immortalis Laus ». Le caractère très ingénieux du texte est sans doute lié à ce rapport hiérarchique, qui pousse le poète à présenter à son protecteur une preuve de son talent.

Au contraire, les auteurs des consolations des Muses en deuil adressées à Alcinde-Brun sont tous des amis ou des proches du défunt. Il arrive d'ailleurs assez souvent que le consolateur fasse partie du même cercle que ceux qu'il console. Puisqu'elle est nécessairement adressée à un interlocuteur, la consolation est le discours par excellence de l'échange poétique, particulièrement dans le cadre de recueils collectifs comme les tombeaux.

\section{La consolation : discours de l'écriture collective}

Le caractère collectif de la consolation dans les recueils poétiques apparaît très clairement dans les tombeaux qui rassemblent les poèmes de plusieurs membres d'une même académie. C'est par exemple le cas de l'une des consolations sur la mort d'Enea Vaini, signée du membre de l'Académie des Ardenti nommé «l'Impuro ». Le poème commence par l'apostrophe suivante : "Quid pia turba gemis Aeneam nuper ademptum?» («Pourquoi pleures tu, ô pieuse foule, la mort récente d'Énée ${ }^{43}$ ?»). Cette « pia turba » se réfère sans doute aux autres membres de l'académie, notamment ceux qui participèrent au recueil.

39 Une nénie à la mémoire de Guido Arnaldo, un membre de l'Accademia Olimpica de Vicence, commence par l'éloge du défunt et non par une lamentation. En plaçant la laudatio au début du poème, le poète peut passer très rapidement de la lamentation et de la consolation à l'exhortation, qui constitue le cœur de ce poème dont le but est d'encourager les fils du défunt à honorer la mémoire de leur père par leur vie :

Vous, jeunes gens, soyez dignes de votre père, quand vous célébrez ses funérailles / Et quand vous rendez les honneurs funèbres, / Retenez vos larmes ; avec une vaillance inaltérable, / Dirigez vos esprits vers les hauts faits de votre illustre père.

Vos Iuvenes Patre digni, dum Iusta parenti

Soluitis et tristes redditis inferias:

Parcite iam lachrimis ; mentes virtute perenni

Dirigite ad magni splendida facta Patris ${ }^{44}$. 
Le poème s'achève sur une évocation du Paradis («domus aurea vestra»), retrouvant ainsi un topos de la consolation chrétienne. L'ordre habituel (éloge, lamentation, consolation) n'a pas entièrement disparu, mais il a été adapté aux fins du poète. Comme la pièce n'est pas signée, il est difficile de déterminer si l'auteur de cette pièce souhaitait s'attirer la protection des fils du défunt, mais la mention de l'Académie Olympique dans le titre du poème et au début du poème laisse penser que le poète vise à encourager les fils d'Arnaldo à s'impliquer comme leur père dans les activités de l'académie.

41 Cet exemple nous encourage à considérer le rôle social et sociable de la consolation dans les tombeaux poétiques. En effet, dans les tombeaux poétiques, la consolation n'est pas exclusivement un discours adressé par un interlocuteur placé en position d'autorité morale ou philosophique à un lecteur placé en posture de disciple. Au contraire, la consolation est souvent l'une des marques de l'échange amical et érudit qui caractérise ces tombeaux poétiques.

42 Cet aspect de la consolation est particulièrement marqué dans les échanges de sonnets qui se font dans ces recueils funèbres.

43 On trouve un exemple frappant de ce phénomène dans les recueils à la mémoire de la comédienne Camilla Rocha Nobili. Cet ouvrage contient cette forme particulière de sonnet en « Risposta » où un poète répond à un autre en reprenant les mêmes rimes que le premier tout en donnant un nouveau sens au poème.

Del Signor Angiolo Trono al Clarissimo Signor Niccolò Boldù.

Signor, cui tanto de la bella estinta

La memoria tormenta à ciascun passo,

Consolate lo spirto afflitto, e lasso,

E sia la doglia dal contrario vinta.

Stassi lei, che piangete, hor la sù accinta

A' Dio, sciolta dal mondo oscuro, e basso,

E dice a voi d'ogni letita casso,

Ch'ella e di stelle, e d'oro, ornata, e cinta.

Ma se'l nobil cadavere honorato

Pur bramate inalzar fin dove arriva

L'alma ben nata à più glorioso stato ;

La penna vostra i pregi suoi descriva.

Cosi potrete incontro al tempo, e al fato

Coronar voi di lauro, e lei d'oliva ${ }^{45}$.

De Messire Angelo Trono au très-illustre Messire Niccolò Boldù.

Messire, qui êtes de la mémoire de la belle défunte tant tourmenté à chaque pas, consolez votre esprit affligé et abattu et que la douleur soit vaincue par son contraire. Elle se tient maintenant, celle que vous pleurez, auprès de Dieu, délivrée $\mathrm{du}$ monde obscur et vil, et elle vous dit, à vous, privé de toute joie, qu'elle est d'étoiles et d'or ornée et ceinte. Mais si pourtant vous désirez élever le noble et honoré cadavre jusqu'où l'âme bien née arrive à l'état glorieux, que votre plume décrive ses mérites. Ainsi vous pourrez, contre le temps et le sort, couronner votre tête de laurier et la sienne de branches d'olivier.

Risposta del precedente del Clarissimo Signor Niccolò Boldù

Cade ogni mio piacer cadendo estinta

La bella DELIA, e perciò l'hore passo

Tormentose, e s'è il senso afflitto, e lasso

Dal duol, anco ragion si rende vinta.

Nè mi giova pensar, che l'alma accinta

A Dio, conosca quanto oscuro, è basso 
Sia per secol nostro, e di letitia casso,

Viva ella di splendor, di gloria cinta.

Nè mi consola ancor che sia honorato

Il raro nome suo fin dove arriva

Ove non giunge de' mortai lo stato.

Respiro sol, ch'i pregi suoi descriva

La vostra penna: questa il tempo, e'l fato

Vice, e inchinarvi fa Dafne, e l'oliva ${ }^{46}$.

Réponse du précédent, du très-illustre Messire Niccolò Boldù.

C'est tout mon plaisir qui disparaît, alors que disparaît la belle Délie, c'est pourquoi je passe les heures tourmentées, mes sens affligés et abattus par la douleur, même ma raison se rend. Et rien ne me sert de penser que l'âme auprès de Dieu apprend à quel point obscure et vile est notre époque, et privé de joie, de penser que cette âme vit ceinte de splendeur et de gloire. Et je ne suis pas non plus consolé par l'idée que son rare nom soit honoré, jusque là où aucun être mortel n'arrive jamais. Je respire seulement, pour que votre plume décrive ses mérites: elle vainc le temps et le destin et fait incliner les têtes de Daphné et de l'olive. du poème est présenté sous les traits d'un amant affligé que Boldù invite à sécher ses larmes. À la fin du huitain, le poète représente la défunte dans son apothéose au ciel. Cependant, dans le sizain, le poète rappelle que son destinataire est poète, pour développer un autre type d'argumentation. L'auteur du sonnet propose à son ami de prendre la plume pour immortaliser les mérites de la défunte, mais également ses propres talents de poète, par le biais de la référence au laurier d'Apollon et à l'olivier d'Athéna. Le laurier est associé à Laure par Pétrarque dans le dernier tercet du sonnet CCXXX du Canzoniere ${ }^{47}$, mais il est également évoqué par Horace dans ses odes ${ }^{48}$. Le poète oppose ici la couronne du poète (le laurier) à celle de la jeune vierge, consacrée par l'arbre de la vierge protectrice d'Athènes.

Le sonnet en réponse de Niccolò Boldù constitue une riposte à cette consolation. Il commence par se présenter comme un être inconsolable, car la cause de son bonheur lui a été enlevée. Il oppose par exemple le vers "Dal duol, anco ragion si rende vinta " à celui qu'avait écrit Angiolo Trono («E sia la doglia dal contrario vinta.»). Il repousse donc l'image de l'apothéose de Délie, ainsi que l'argument selon lequel le nom de sa bienaimée sera célébré éternellement. En revanche, dans le dernier tercet, le poète évoque l'espoir que la plume de son ami vaincra le temps et fera incliner les têtes de «Daphné et de l'olive", les deux couronnes évoquées à la fin du premier poème, donnant clairement aux images du laurier et de l'olive une interprétation métapoétique.

À travers un jeu avec la forme de la risposta, ces sonnets mettent en scène l'échec des arguments traditionnels de la consolation, l'ascension au ciel de la défunte après sa mort et l'immortalité conférée à celle-ci par la poésie. En revanche, les poèmes se terminent tous deux par un appel à prendre sa plume. En fin de compte, ces sonnets ressemblent bien plus à des compliments adressés à un camarade poète qu'à une consolation adressée à un ami. Pourtant, ils prennent racine dans la tradition du discours de consolation. Bien sûr, le ton de ces poèmes est lié à la forme choisie, car la risposta est nécessairement un jeu avec un autre poète, qui implique la rivalité ou la flatterie. Toutefois, il semble que ces poèmes témoignent d'une logique plus générale qui est à l'œuvre dans les tombeaux poétiques : dans ces recueils collectifs, le deuil poétique est avant tout un moment de rassemblement et d'émulation. Le défunt est devenu, quant à lui, la matière du poème, un sujet de conversation entre les poètes. 
logique du rassemblement est également présente dans des sonnets qui ne prennent pas la forme de la réponse. L'on peut prendre comme exemple les sonnets adressés à Brun-Cléante par son ami Nicolas Faret dans les Muses en deuil.

Mesme encor aujourd'huy, loin de notre sejour

Mille Cygnes nouveaux se rengent à l'entour

Pour former un concert de complaintes funebres ${ }^{49}$.

Ici encore, l'argument final est la référence au concert des poètes qui se rassemblent autour de la défunte pour célébrer sa mémoire. Un autre sonnet du même Faret se présente comme une consolation qui s'achève, comme les sonnets italiens déjà évoqués, sur une métaphore du tombeau poétique.

Berger, fay donc tarir les ruisseaux de tes pleurs,

Car tu sçais qu'au Printemps on cueille ainsi les fleurs

Dont nous voulons parer l'Autel de nostre Temple ${ }^{50}$.

49 Ces «fleurs" qui parent l'autel de l'église peuvent être interprétées comme les ornements poétiques dont est composé le recueil, dans la mesure où l'anthologie est, étymologiquement, un «choix de fleurs». De façon assez topique, le recueil poétique lui-même est présenté comme un temple, mais il faut noter que ce monument est présenté comme une œuvre collective ( notre temple »). Dans ce contexte de poésie collective, la visée de la consolation semble parfois être l'émulation et la virtuosité davantage que sa valeur thérapeutique.

\section{Conclusion}

50 L'étude des consolations en vers dans les tombeaux poétiques européens du début du XVII ${ }^{e}$ siècle révèle en fin de compte une grande diversité de pratiques d'écriture. Il apparaît que, loin d'être clairement distinguée d'autres formes de commémoration comme l'éloge ou la lamentation, la consolation accompagne, inclut ou conclut ces discours. Cela nous encourage à définir les discours funèbres par la pratique autant que par la théorie et à considérer les formes de la commémoration des défunts comme un tissu continu de pratiques plutôt que comme des catégories bien distinctes. Ce fait a été noté par les théoriciens de la Renaissance, mais il est également vérifié par la pratique, puisque l'on constate que la consolation peut être intitulée "épitaphe» ou «nénie ». De même, on observe qu'elle peut prendre une multiplicité de formes, des plus brèves, souvent associées aux formes lapidaires comme l'épitaphe ou l'épigramme, aux plus longues. Cette indétermination formelle semble mettre en péril toute tentative de définir la consolation en tant que genre. Cependant, la solution est de considérer qu'elle est non pas un genre défini par des critères formels, mais plutôt une forme de discours qui se caractérise par une situation d'énonciation particulière, celle où un locuteur s'adresse à son destinataire pour le consoler d'une perte ou d'un chagrin (dans le cas évoqué dans cet article, un décès). Dans ce cadre-là, un grand nombre de variations sont possibles, et c'est d'ailleurs dans cet exercice de variatio que les poètes cherchent à briller, puisqu'il démontre leur capacité à s'adapter à la condition de leur interlocuteur ainsi que leur talent pour l'invention formelle. En effet dans les recueils funèbres, la consolation peut fournir l'occasion d'un véritable échange - pour ne pas dire un concours - entre des poètes d'un même cénacle. Forme implicitement dialogique, la consolation occupe dès lors une place de choix dans ces lieux de sociabilité littéraire que sont les tombeaux collectifs. Ce constat nous invite à 
considérer non plus exclusivement la valeur philosophique de la consolation, mais sa valeur sociale, voire sociable, sans doute dérivée de son origine épistolaire. Objet d'émulation et de reconnaissance entre pairs, la consolation devient une sorte de pierre d'angle du temple poétique que les poètes érigent collectivement.

\section{NOTES}

1. Plutarque, Consolation à Apollonius, traduction de J. Defradas et J. Hani dans Plutarque, CEuvres morales, vol. II, Paris, Les Belles Lettres (CUF), 1985, p. 41. Plutarque rappelle ici à son lecteur un vers de Prométhée enchaîné, bien que la citation soit inexacte.

2. Pour Lucrèce : voir dans le présent DOSSIER d'Exercices de rhétorique, S. Luciani, « Lucrèce et la tradition de la consolation ». Le livre III du De natura rerum de Lucrèce contient une diatribe contre la mort qui peut être considérée comme une lecture de la Lettre à Ménécée. Voir à ce sujet B. Price Wallach, Lucretius and the diatribe against the fear of death, De rerum natura, III 830-1094, Leyde, E. J. Brill, 1976.

Pour Ovide : voir Ovide, Pontiques, 4, 11 (J. André éd., Paris, Les Belles Lettres, 1993).

Pour Stace : voir Stace, Silves, 2, 1 et 2, 6 ; 3, 3 ; 5, 1 (tome I, C. Moussy éd., Paris, Les Belles Lettres, 1992).

Pour la Consolatio ad Liviam, aussi connue sous le titre Epicedion Drusi : voir Pseudo-Ovide, Epicedion Drusi. Consolation à Livie. Élégies à Mécène. Bucoliques d'Einsiedeln, J. Amat éd., Paris, Les Belles Lettres, 1997.

3. Dans les élégies d'Ovide, Amours, 3, 9 sur la mort de Tibulle. On peut aussi citer la quatrième élégie de Properce, Élégies, 4, 11(D. Paganelli éd., Paris, Les Belles Lettres, 1964).

4. Pour Horace : Horace, Odes, 2,9 (Fr. Villeneuve éd., Paris, Les Belles Lettres, 1997).

Pour Martial : Martial, Épigrammes, 5, 37 (H. J. Izaac éd., Paris, Les Belles Lettres, 1969-1973).

5. Pour une étude plus approfondie, voir notre thèse de doctorat: "Les recueils de poésie funèbre imprimés en Italie, en France et dans les Îles britanniques (1587-1644) ", soutenue à l'université Paris-Sorbonne le 18 janvier 2016.

6. G. Chiecci, La parola del dolore, primi studi sulla letteratura consolatoria tra medioevo e umanesimo, Rome et Padoue, Antenore, 2005.

7. Jules-César Scaliger, Poetices libri septem, Livre III, chapitre 121 (notre traduction). Cf. JulesCésar Scaliger, Poetices libri septem, Lib. I, Cap. L, "Threni et hymenaei et elegiae; alia ", dans Poetices libri septem, Sieben Bücher über die Dichtkunst, übersetzt, eingeleitet und erläutert von Luc Deitz und Gregor Vogt-Spira ; unter Mitwirkung von Manfred Fuhrmann, Stuttgart-Bad Cannstatt, FrommannHolzboog, 1994-2011, p. 414. Pour le chapitre sur la consolation (trad. Ch. Noille), voir dans le présent numéro d'Exercices de Rhétorique la section ATELIER.

8. Contrairement aux hymnes, qui visent à louer les dieux. Voir Ménandre le rhéteur, Menander Rhetor, trad. D. A. Russell et N. G. Wilson, Oxford, Clarendon Press, 1981, p. 325, commentaire de 413.5-414.30, Paramuthètikos.

9. In anniversarium Henrici Magni obitus diem, lacrymae collegii flexiensis regii societatis Iesu, La Flèche, Jacques Rezé (imprimeur du roi), 1611.

10. "In mortem Henrici Magni Naenia », dans In anniversarium Henrici Magni obitus diem, op. cit., p.130-131. L'auteur est anonyme, comme il arrive souvent dans ces compositions de 
circonstance. Dans cet article, quand l'auteur n'est pas mentionné dans le corps du texte ou en note, c'est qu'il n'a pas signé. Toutes les traductions de ces pièces sont nôtres.

11. Ibid.

12. Pour Stace, voir supra note 2. Pour l'épicède de Politien sur la mort d'Alibera degli Albizzi, voir Due poemetti latini : elegia a Bartolomeo Fonzio : epicedio di Albiera degli Albizi, F. Bausi éd., Rome, Salerno, 2003.

13. J. Esteve-Forriol, Die Trauer- und Trostgedichte in der römischen Literatur, untersucht nach ihrer Topik und ihrem Motivschatz, Munich, A. Schubert, 1962, p. 78-107.

14. "Naenia », Clarissimi viri Simeonis Provencherii, medici regii et Senonensis tumulus, Sens, George Niverd (ou Nyverd), 1617, p. 10-11.

15. Epitaphs upon the untymelie death of W.[illiam] Michel, Aberdeen, Edward Raban, 1634, D4r.

16. M. R. B., " An epitaph, or consolatorie epistle upon the death of the sayd young man, written to his mother by M.R.B., preacher of the Evangel », ibid.

17. Billon, "Nicolao e Verduno protopraesidi amplissimo, de clarissimae heroinae Carolae du Guai conjugis carissimae obitu, Consolatio ", dans Jean d'Artis, [Tombeaux, oraison funèbre, consolations et poèmes divers]. s.l.n.d. [1621], p. 136. - Notre traduction ne peut évidemment rendre l'anagramme.

18. Carmina sex Academicorum ingeniosorum Salodii in obitu ill.mi Iacobi comitis Valmaranae, et Equitis Santi Jacobi Hispaniarum declarati, Vicence, Domenico Amadio, 1612. Le poème cité est de Franco Franchi, prince des Ingegnosi, A2r.

19. Ibid. : "Quid ruis in lacrymas, quod moeror corda fatiga[t] / Pars animae genitrix, dimidiumque meae ?»

20. Ibid. : « Me si Mater amas fletus compesce fluentes, / Cordas languenti solve dolore precor. »

21. Ibid. : " Haud moriar quicquid mortale in funere linquens, / Vivam, abiisse magis, quam periise puta. »

22. Les Muses en deuil, en faveur du sieur Brun, sous le nom de Cléante. Pour la mort de son Alcinde, Paris, Toussaint Du Bray, 1620, p. 46-51.

23. Tombeau dressé à la bienheureuse memoire du R. P. Mre Claude de Montigny, Prestre, \& Superieur de la Ste Congregation de l'Oratoire de Jesus, en la ville d'Orleans, où il deceda le 16. Jour de Novemb. 1624. Par M. J. Le V. [...], Paris, Pierre de Bresche, 1625, p. 127-130.

24. Indubitablement, les choix poétiques sont également influencés par l'identité des auteurs eux-mêmes, mais le fait que les pièces soient souvent non signées ne permet pas une analyse exhaustive à ce sujet.

25. G. Chiecchi affirme que l'œuvre d'Ambroise ne met pas simplement en avant la destruction de la douleur par la considération de la résurrection, dans la mesure où elle commence par une réelle prise en compte de la douleur et du deuil (concessio doloris), pour considérer ensuite la résurrection. Comme on l'a vu, sans cette prise en considération initiale de la douleur, le discours consolatoire courrait le risque de tout simplement disparaître, puisque la croyance en la résurrection rendait vaine toute peur de la mort, fondée qu'elle était sur la certitude que le Christ en ressuscitant avait vaincu la mort. Selon lui, Ambroise serait ainsi parvenu à dépasser le paradoxe d'un genre qui semblait à première vue inadapté à la religion chrétienne et lui aurait donné une force nouvelle grâce à la croyance en la résurrection. Voir G. Chiecchi, "Sant'Ambrogio e la fondazione della consolatoria cristiana », dans La parola del dolore, op. cit., p. 3-46.

26. Mausolee, ou Temple d'honneur, Dressé à l'éternelle memoire de Messire Florimond d'Ardres, baron de Frican, Malberg, Odun, \&c [...]. Par le Chevalier de Lescale [Adam Scaliger], \& les plus beaux Esprits de l'Univers, Paris, Robert Daufresne, 1622.

27. S. Dumas, «Consolation à Monsieur le Duc de Deux-Ponts \& Madame de Rohan sur la mort de Madame la Duchesse de Deux-Ponts ", Le Tombeau de tres haute, tres illustre et tres vertueuse Princesse Catherine de Rohan, duchesse de Deux-Ponts par L. F., Paris, Jean Jannon, 1609, p. 49-52.

28. Ibid. 
29. Catherine de Rohan, op. cit., p. 50.

30. A. Tarrête, «Remarques sur le genre du dialogue de consolation à la Renaissance ", Réforme, Humanisme, Renaissance, $\mathrm{n}^{\circ}$ 57, déc. 2003, p. 133-152.

31. Catherine de Rohan, op. cit., p. 41.

32. Par exemple : « Epigramma. Matrem suam lacrymantem Iacobus moriens consolatur », Carmina sex Academicorum ingeniosorum Salodii, op. cit., A2r, ou encore ibid., "Ad Honoratiss. Matrem Consolatio " C4v; "L'ombra di G. G. la madre Maddalena consola », in Beatae Mariae Magdalenae lachrymae, in obitum Guglielmi Grey, Domini Arthuri Grey Baronis de Wilton, aurea Periscelidis Equitis Clarissimi, Filii natu, Oxford, Joseph Barnes, 1606, F4v.

33. G. B. Basile, "Consola la maestà del Re », in Relatione della pompa funerale che si celebrò in Napoli nella morte della serenissima reina Margherita d'Austria, Naples, Tarquinio Longo, 1612, p. 35 ; A. Spina, «Consola la Maestà del Re », ibid., p. 36.

34. Voir G.J. Vossius, Poëticarum Institutionum Libri Tres, III, 21, § 12 (incipit), dans Vossius, Tractatus philologici de rhetorica, de poëtica, de artium et scientiarum natura ac constitutione, Amsterdam, P. et J. Blaeu, 1597, p. 167 : « At illud non invenustum, alloqui tumulum, vel urnam, quae continet vita functum : vel Academiam, curiam, forum, patriam, quae amisit : vel religionem, aut fidem, si sacerdos erat, vel vir pietate eximius. "

35. «De parricidio Henrici IIII (...) Elegia consolatoria ad Galliam», in Recueil de diverses poésies sur le trespas de Henri le Grand très chrestien roy de France et de Navarre. Et sur le sacre et le couronnement de Louis XIII, son successeur, Paris, Robert Estienne, [1611], p. 62 et « Providentia divina Galliam super sui regis interitu consolatur. Hac ode tricolo tetrastropho », ibid., p. 287.

36. "Consolatio Galliae in funere Richelaei », in Monumentum eucharisticum P. M. Eminentissimi Joannis Armandi du Plessis Cardinalis Ducis Richelii. A Collegio Lugdunensi SS. Trinitatis Societatis Jesu Dedicatum, Lyon, Jacques Roussin, s.d. [1643], p. 43.

37. « Bolonia llorando consolada ", in Il funerale fatto dal Senato di Bologna all'illustriss.o et ecc.mo sig.r D. Carlo Barberino generale di S. Chiesa, Bologne, héritier Benacci, 1630, p. 31.

38. "Ad moestos Britones consolatorium", in Luctus posthumus sive erga defunctum Henricum Walliae principem, Magdalensium officiosa pietas, Oxford, Joseph Barnes, 1612, F3r.

39. Relazione della pompa funebre celebrata dalla città di Viterbo per la morte di [...] Enea Vaini, suo governatore, a 9 di maggio 1633, Viterbe, Diotallevi, 1633, p. 43.

40. "Gens Baleonia Perusiam consolatur", in L'Apparato funerale fatto in Perugia nell'essequie dell'illustrissimo Adriano Baglioni disteso in narratione dal distratto academico insensato, Rome, Cesare Scaccioppa, 1624, p. 55.

41. " Ad Academiam Carmen consolatorium », in Justa funebria Ptolemaei Oxoniensis T. Bodleii, Oxford, Joseph Barnes, 1613, p. 34.

42. « Ad Excellentiss. I.V.D.D. Iulium à Vulpe patronum eximium. In obitu Illustriss. D. Co. Aloysii Porto grati clientis consolatio ", dans Oratione funebre ne l'essequie del'ill.mo sig. conte Luigi Porto, composta dal p. d. Francesco Zoia, et recitata da l'istesso ne la chiesa di S. Lorenzo de' frati minori in Vicenza il di 4 aprile 1612, Vicence, Francesco Grossi, [1612], p. 40.

43. Dans «In funere eiusdem consolatio impuri accademici Ardenti », dans Relazione della pompa funebre celebrata dalla città di Viterbo per la morte di... Enea Vaini, suo governatore, a 9 di maggio 1633, Viterbe, Diotallevi, 1633, p. 43.

44. Ibid., p. 8.

45. Le funebri rime, di diuersi eccellentissimi autori, in morte della signora Camilla Rocha Nobili Comica Confidente, detta Delia, raccolte da Francesco Antonazzoni Comico Confidente detto Ortensio, Venise, Ambrosio Dei, 1613, p. 123.

46. Ibid., p. 123.

47. « Non lauro o palma, ma tranquilla oliva / Pietà mi manda, e 'l tempo rasserena, / e 'l pianto asciuga, et vuol anchor ch'i' viva. » : « Ni palme, ni laurier, mais la tranquille olive / Pitié m'envoie, et le temps 
rassérène, / Et les pleurs sèche, et veut encore que je vive ", dans Pétrarque, Chansonnier, Rerum Vulgarium Fragmenta, G. Savoca et G. Genot éd., Paris, Les Belles Lettres, 2009, p. 324-325.

48. Horace, Odes, I, 7.

49. Les Muses en deuil, op. cit., p. 27.

50. Ibid., p. 26.

\section{AUTEUR}

\section{PAULE DESMOULIÈRE}

Université Paris Sorbonne (OBVIL - Observatoire de la vie littéraire) 